\title{
A high power laser facility to conduct annealing tests at high temperature
}

M. Minissale, ${ }^{1, \text { a) }}$ A. Durif, ${ }^{2}$ P. Hiret ${ }^{3}$ T. Vidal, ${ }^{3}$ J. Faucheux, ${ }^{4}$ M. Lenci, ${ }^{5}$ M. Mondon, ${ }^{5}$ G. Kermouche,${ }^{5}$ Y. Pontillon, ${ }^{4}$ C. Grisolia, ${ }^{2}$ M. Richou, ${ }^{2}$ and L. Gallais ${ }^{3, b}$ )

1) Aix Marseille Univ, CNRS, PIIM, Marseille, France

${ }^{2)}$ CEA, IRFM, F-13108 Saint-Paul-Lez-Durance, France

${ }^{3)}$ Aix Marseille Univ, CNRS, Centrale Marseille, Institut Fresnel, France

4) CEA, DEN/CAD/DEC/SSA3C, Saint-Paul-lez-Durance, France

${ }^{5)}$ Ecole Nationale Supérieure des Mines de Saint-Etienne, LGF, CNRS UMR 5307, Saint-Etienne, France

The knowledge of materials properties and their behavior at high temperatures is of crucial importance in many fields. For instance annealing phenomena occuring during the thermomechanical processing of materials, such as recrystallization, have long been recognized as being both of scientific interest and technological importance. Different methods are currently used to study annealing phenomena and submit metals to heat loads. In this work, we present the design and the development of a laser-based facility for annealing tests. This experimental setup enables studies at the laboratory scale with great flexibility to submit samples to various spatial and temporal heating profiles. Due to the possibility to have optical access to the sample, laser heating can be combined to several non-contact diagnostics such as infrared imaging to control and analyse the temperature gradients. As case study, we present a set of experiments performed to study the recrystallization kinetics of tungsten. We demonstrate that samples can be heated linearly with heating rate up to $\sim 2000$ $\mathrm{K} / \mathrm{s}$, at temperatures above $2000 \mathrm{~K}$, for seconds or hours, with typical errors in the temperature measurement of around $1 \%$ that depend mainly on the determination of sample emissivity. Such studies are of crucial interest in the framework of nuclear fusion, since the ITER nuclear reactor will operate with a full-W divertor.

Keywords: ITER, Laser heating, Recrystallization, Tungsten

\section{INTRODUCTION}

Annealing phenomena occuring during the thermomechanical processing of materials, such as recrystallization, have long been recognized as being both of scientific interest and technological importance. Such phenomena can occur in all types of crystalline materials, on rocks and minerals during the natural geological deformation, on ceramics during the technical processing, and so on ${ }^{1}$. However, annealing phenomena have been most widely studied in metals. The requirements of industry have pushed to perform more and more accurate and quantitative studies and to develop physically-based models which can be applied to metal-forming processes so as to control, improve and optimize the microstructure and texture of products. Moreover, such studies have allowed to understand the evolution of metals when submitted to specific physical-chemical conditions. Metalworking and heating procedures have been practised since thousands of years, but only recently we gained some understanding of the structural changes induced by annealing phenomena ${ }^{1,2}$.

Different methods are currently used to study annealing phenomena and submit metals to heat loads: resistance heating (i.e. electropulsing treatment ${ }^{3-6}$, conventional furnace system ${ }^{7}$, inductive oven ${ }^{8}$, electron gun ${ }^{9,10}$, rapid immersion cycles in a salt bath ${ }^{11}$, pulsed and con-

\footnotetext{
a) email adress: marco.minissale@univ-amu.fr

b) email adress: laurent.gallais@fresnel.fr
}

tinuous laser exposure ${ }^{12-14}$. It is well known that the heating rate has a strong effect on the recrystallization process and on the morphological evolution during annealing and recovery ${ }^{15,16}$. Low heating rate can result in nucleation of new grains during the heating stage that can then accelerate the recrystallization process. It can also play on recovery - decrease of the intragrain stored energy - and thus slow-down the recrystallisation process. Therefore playing on the heating rate is one way to control/optimize the microstructure of metals after annealing. Despite this interesting feature, it means that the measurement of recrystallization kinetics during annealing treatment may be unfortunately heating-rate dependent. To overcome this difficulty the highest available heating rate should be used. The different techniques listed above allow to induce annealing in a broad timescale domain, from the from fractions of seconds in the case of pulse laser system to the hour in the case of conventional furnace system. In addition to the annealing timescale, other factors, such as the sample size or constraints given by the experimental setup, could make suitable one technique rather than another. In particular, the use of the laser heating is convenient when a remote sample processing is required. Actually the laser-matter interaction can be efficiently used to bring material in extreme conditions of temperature and pressure at the laboratory scale. Such conditions can be efficiently applied to modify the material properties with an accurate control of the energy deposition in both space and time.

In this paper, we describe the design and development of a high power laser facility to submit materials to ex- 
treme heat loads. We present some possible applications of the experimental facility, in particular focusing our attention on the laser-induced annealing of tungsten (W). Annealing phenomena of $\mathrm{W}$ are of crucial interest in the framework of nuclear fusion. The fusion reaction could become a viable way to generate electricity. To perform this reaction, reactors confine magnetically plasma in a vacuum chamber. However, plasma confinement is imperfect and energy losses are directed mainly on the lower part of the vessel called divertor. The ITER (International Thermonuclear Experimental Reactor) nuclear reactor will operate with components made up of chains of $\mathrm{W}$ monoblocks bonded to a $\mathrm{CuCrZr}$ tube, with a pure $\mathrm{Cu}$ interlayer in between ${ }^{17,18}$. These components will face to heat flux up to $20 \mathrm{MW} / \mathrm{m}^{219}$. Due to high heat flux, strong temperature gradients are generated on a thickness of $7 \mathrm{~mm}$, leading to extreme temperature values from $2300 \mathrm{~K}$ at the loaded surface to $800 \mathrm{~K}$ near the cooling tube ${ }^{20}$. Such temperatures are large enough to alter the tungsten microstructure causing recrystallization and mechanical properties losses and then damages such as macro cracks in the material ${ }^{21,22}$. Recrystallization is the phenomenon that consists in the replacement of a deformed microstructure by new grains, that are virgin of dislocations or subgrain boundaries. The usual consequence of recrystallization is the decrease of mechanical properties. In the case of tungsten, recrystallization could also affect in a negative manner the Brittle to Ductile Transition Temperature ${ }^{23}$. The deterioration of the thermal shock resistance ${ }^{24}$ may lead to the premature development of macro-cracks on these components during transient divertor re-attachment in ITER ${ }^{25}$. For such reason it is mandatory to study in detail the recrystallization kinetics of the $\mathrm{W}$.

\section{EXPERIMENTS AND METHODS}

\section{A. Laser-induced heat loads configuration}

We propose in this work to use laser remote heating to generate heat loads and to conduct annealing tests at high temperature on materials for plasma facing components. We will restrict the discussion about the experimental configuration and our examples to tungsten samples even if the technique can be applied to other materials of interest. Based on the optical properties of tungsten, a laser in the near infrared spectral range is optimal for this work since we can benefit both from the relatively low total (diffuse+specular) reflectivity (between 50 and $60 \%{ }^{26}$ ) to have good coupling of laser light in the material and from available high power lasers (Laser diodes or Ytterbium fiber lasers). We have worked with a high power Ytterbium fiber laser emitting at a wavelength of $1080 \mathrm{~nm}$, with a corresponding reflectivity of $62 \%^{26}$.

Thermal annealing tests require homogeneous heating in the whole sample for correct interpretation of results, as a minimal time for ramping up and down the temperature. We have therefore firstly conducted thermal simulations in order to find the best compromise on sample size, that needs to be sufficient enough to analyse the microstructure, but needs to be small enough to minimize the thermal gradients since the laser radiation is absorbed on the surface. Moreover a reasonable amount of power is required to reach the required temperature (few hundred watts). Additionally, for practical purposes (cutting of samples from a $\mathrm{W}$ part), the samples have a parallelepiped geometry and the beam has a Gaussian spatial profile. This dimensioning of the experiment has been achieved by numerical simulations with COMSOL Multiphysics Software (Finite Elements Method), with thermal parameters extracted from ITER Database. Based on the described constraints, we have worked with samples of few $\mathrm{mm}^{3}$ : parallelepipeds of $4 \times 4 \times 5 \mathrm{~mm}^{3}$ (purity $99.95 \mathrm{wt} \%$ ) with laser-induced heat loads applied from both sides to ensure temperature homogeneity (Fig. 1).

Figures/Fig_simu_W_3D.pdf

FIG. 1. Numerical simulations of laser heating on $4 \times 4 \times 5 \mathrm{~mm}^{3}$ $\mathrm{W}$ samples. (left) Incoming heat flux on opposite sides of the samples. The laser intensity distribution is Gaussian, centered on the sample faces : (right) Temperature isotherms obtained in steady state regime.

In this configuration the calculated temperature gradients, difference of temperature between the center of surface exposed to the laser (maximum) and sample edge (minimum) has been calculated for different steady state annealing temperatures. The gradient is below $20 \mathrm{~K}$ for a temperature of $2000 \mathrm{~K}$ (see example on Fig. 1) and below $10 \mathrm{~K}$ for a temperature of $1700 \mathrm{~K}$. However during the transient heating and cooling the gradient can be higher. This is illustrated in Fig. 2 where we have simulated a typical sequence under consideration: a linear increase at $200 \mathrm{~K} / \mathrm{s}$ (on faces exposed to the laser flux) up to 2300 $K$, followed by a plateau of $10 \mathrm{~s}$ and then cooling back 
to the ambient temperature.

In such a case the temperature gradient reaches $40 \mathrm{~K}$ in the transient sequence, versus $20 \mathrm{~K}$ during the plateau. This corresponds to temperature deviations in the sample below $2 \%$ compared to the temperature setpoint.

\section{B. Experimental setup}

The experimental setup, called ChauCoLase (Chauffage Controlé par Laser, laser controlled heating in english), is based on a high power CW Ytterbium fiber laser (SPI laser Qube 1500) which can deliver 1500 $\mathrm{W}$ of maximum power with a monomode laser beam and with typical rising time of few microseconds. The main beam can be splitted in several beamlets with the appropriate optical components, that can be sent through laser windows in a dedicated vacuum chamber with a base pressure of $2 \times 10^{-2}$ mbar. As shown in Fig 3 , the sample of interest is placed at the center of the experimental chamber on a sample holder made of high temperature resistant materials such as graphite. Laser beam diameter on the sample surface was set to 4 $\mathrm{mm}$ diameter (diameter at $1 / \mathrm{e}^{2}$ ). Only one side of the sample is in contact with the holder to minimize thermal losses. Numerical simulations have been performed in this configuration and the sample holder has negligible effects on the thermal gradients in the sample.

The optical instrumentation around the sample is a key point to monitor and control the temperature, therefore optical diagnostics such as thermal imaging and optical pyrometry have been implemented through differ-

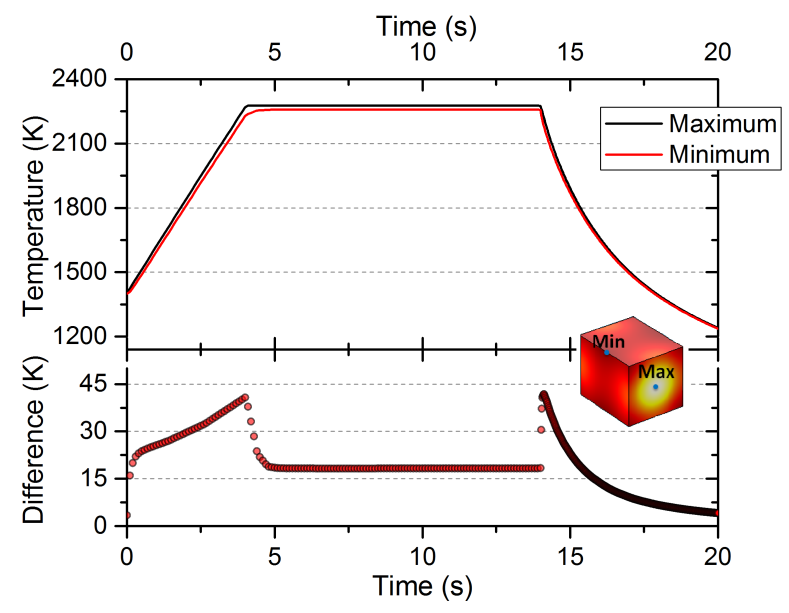

FIG. 2. Simulation of a laser-heating sequence on a $4 \times 4 \times 5 m^{3} \mathrm{~W}$ sample, in the configuration described in the previous figure. The laser power is adjusted to obtain a linear temperature increase at $200 \mathrm{~K} / \mathrm{s}$, followed by a temperature plateau. The laser flux is shut-off at the end of the plateau and the sample cooled back to the ambient due to radiation losses. The maximum and minimum temperatures on the sample are plotted in the top panel while the bottom panel shows the amplitude of the temperature gradient.

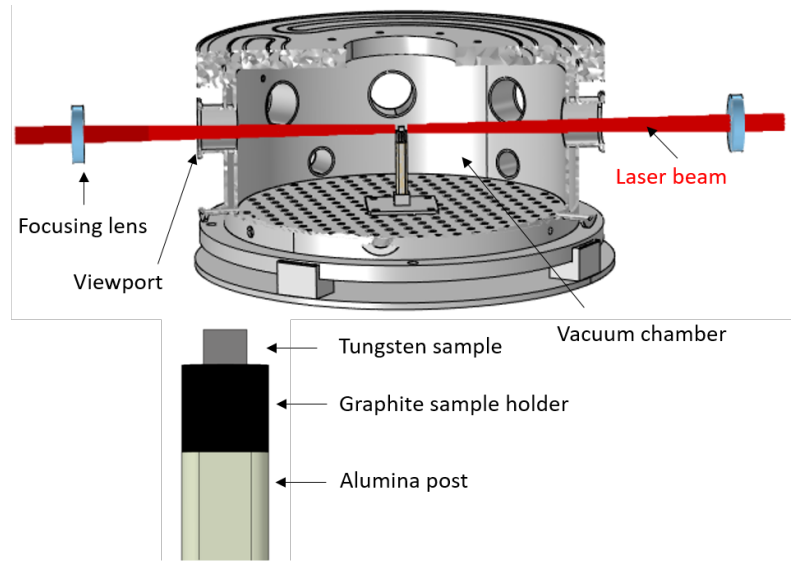

FIG. 3. Schematic of the experimental chamber and sample holder. The sample is placed at the center of a chamber equipped with 8 viewports for laser beams and optical instrumentation.

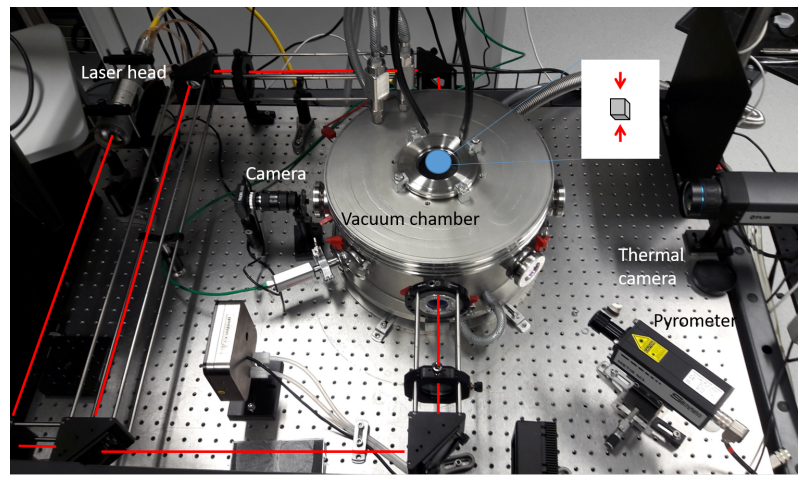

FIG. 4. Top-view picture of the ChauCoLase setup.

ent viewports of the experimental chamber. Other ports are available for thermocouple vacuum feedthroughs or pressure measurements. A picture of the experimental configuration is given on Fig. 4.

The temperature of the sample is monitored with several diagnostics: a type-C thermocouple, an infrared camera (FLIR, model A655sc) working at 7-14 $\mu \mathrm{m}$ in the 233-2273 K temperature range, several optical pyrometers operating in the near infrared spectral range, through an anti-reflective coated window in the spectral range of interest. In the present work, we employed a monochromatic (MC) pyrometer (SensorTherm, model Metis M313) working at $1.27 \mu \mathrm{m}$ in the $873-3800 \mathrm{~K}$ temperature range, a bichromatic (BC) pyrometer (SensorTherm, model Metis H322) working at 1.45-1.65 $\mu \mathrm{m}$ in the $823-1673 \mathrm{~K}$ temperature range. All pyrometers used have a high optical resolution: the measurement spot size on the sample has a diameter below $1 \mathrm{~mm}$. An integrated laser pointer ensures that the measurement spot size is positioned at the center of the sample surface, where the laser spot is centered. The thermal camera can also be used to observe the temperature gradient on the stud- 
ied sample, as a CMOS camera operating in the visible range for sample monitoring during the experiments. A PID (Proportional, Integral, Derivative) feedback loop has been implemented to ensure well-controlled heating ramps and stable temperature set-points. It is based on the temperature measured with the pyrometer that is used as the input signal of a PID program controller (Sensortherm Regulus) that controls the laser power output via a $0-10 \mathrm{~V}$ analog output. We will discuss about the performances of this system in the next section.

\section{EXPERIMENTAL PERFORMANCES}

\section{A. Temperature calibration}

As already said in the previous section, the temperature is measured by different diagnostics. The use of each diagnostic is suitable for different applications and materials. The optical diagnostics are ex-situ methods and they do not require any constrains in terms of sample structure. On the other hand, due mainly to the different working spectral range, each optical diagnostic is appropriate for a specific material: the use of the MC pyrometer is suitable for tungsten sample since the working wavelength is in the $\mathrm{X}$-point region (emissivity does not vary as a function of temperature) of $\mathrm{W}^{27,28}$. This represents a major advantage since, for a given $\mathrm{W}$ sample, the emissivity determined at room temperature can be used in a large temperature range. The main advantage of the $\mathrm{BC}$ pyrometer is that it can be used for all types of samples, but emissivities $(\epsilon)$ at $1.45-1.65 \mu \mathrm{m}$ (and their ratio) have to be known (or determined) for each temperature. The infrared Camera is suitable only for high-emissivity materials (e.g. graphite) and it allows to determine temperature gradient on the studied sample without accurate knowledge on the absolute temperature in case of W. Finally, the thermocouple can be only used for specific samples equipped with a hole where the thermocouple is inserted for calibration purposes.

Since the main part of experiments has been performed on $\mathrm{W}$ samples, in the present work we focus in particular on the calibration of the MC pyrometer. The MC pyrometer was firstly calibrated through a black body source up to $1473 \mathrm{~K}$. In the present case $\epsilon$ was fixed to 0.99 and the deviation between black body source and pyrometer measurement was lower than $2 \mathrm{~K}$. Secondly, we compared $\mathrm{MC}$ and $\mathrm{BC}$ pyrometer measurements. We have varied emissivity values for BC pyrometer $\left(\epsilon_{1.45 \mu m}, \epsilon_{1.65 \mu m}\right.$, and $\left.\epsilon_{1.45 \mu \mathrm{m}} / \epsilon_{1.65 \mu \mathrm{m}}\right)$ while keeping the same value for the MC $\left(\epsilon_{1.27 \mu m}\right.$ was fixed in the $0.25-0.35$ range), trying to minimize the deviation between the temperature measurements of the two pyrometers. We have used the starting emissivity values from ${ }^{28}$. The temperature differences between pyrometers was minimized for $\epsilon_{1.27 \mu m}=0.32$ with a minumum of $\pm 25 \mathrm{~K}$ in the $873-1673 \mathrm{~K}$. One can note that 0.32 is only strictly valid for the used sample, since emissivity can drastically change as a function of sur-

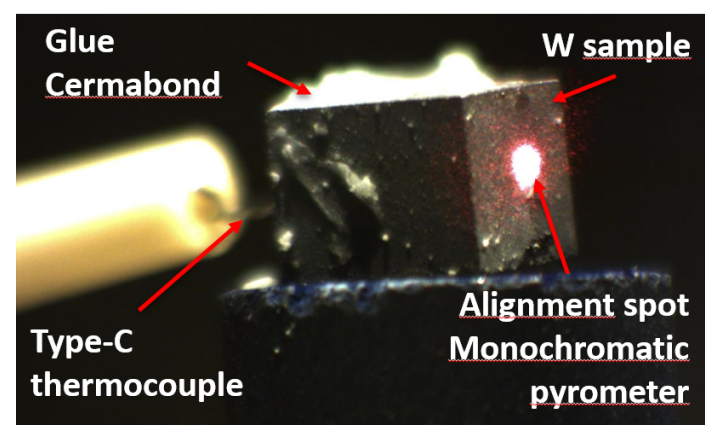

FIG. 5. Detail of the experimental configuration employed during temperature calibration.

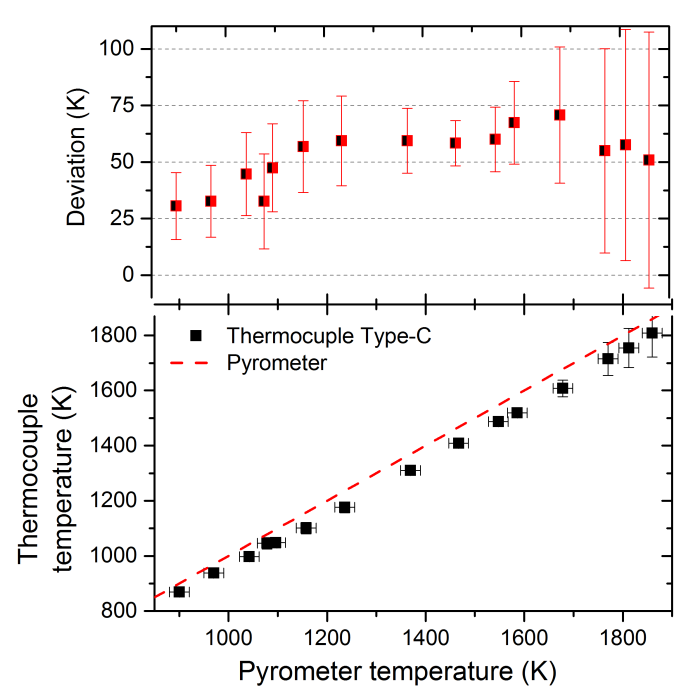

FIG. 6. Temperature measured through a thermocouple type $\mathrm{C}$ and a monochromatic pyrometer $(\lambda \sim 1.27 \mu \mathrm{m})$ on a tungsten sample as a function of temperature, corresponding to different laser power level. The top panel shows the deviation between thermocouple and pyrometer measurements. The emissivity was set to 0.32 .

face state (e.g. oxidation or roughness). MC pyrometer was also calibrated by comparison with type- $\mathrm{C}$ thermocouple measurements on the same type of $\mathrm{W}$ sample. The thermocouple was provided by OMEGA, it had a $3 \mathrm{~mm}$ plait ending with a $0.32 \mu \mathrm{m}$ diameter weld joint. It was inserted in a $3 \times 0.4 \mathrm{~mm}$ (depth/diameter) hole and fixed by using a high temperature ceramic adhesive (Ceramabond $^{T M}$ 552, AREMCO), as shown in Fig. 5. We have used the following in-house voltage-temperature calibration for the thermocouple:

$$
\begin{aligned}
T= & -1.83769+82.31452 \cdot V-9.92347 \cdot V^{2} \\
& +2.04927 \cdot V^{3}-0.25700 \cdot V^{4}+0.01945 \cdot V^{5} \\
& -8.91613 \cdot 10^{-4} \cdot V^{6}+2.42492 \cdot 10^{-5} \cdot V^{7} \\
& -3.59821 \cdot 10^{-7} \cdot V^{8}+2.24361 \cdot 10^{-9} \cdot V^{9}
\end{aligned}
$$

Fig. 6 shows the comparison between temperature of W sample (bottom panel) measured via the thermocou- 
ple and the MC pyrometer as a function of power. In the top panel, we have plotted the deviation $\mathrm{T}_{M C p y r 0^{-}}$ $\mathrm{T}_{\text {thermocouple }}$ vs the temperature. The mean value of the deviation is $\sim 50 \mathrm{~K}$ with a maximum value of $75 \mathrm{~K}$ at $1680 \mathrm{~K}$. A positive value of such deviation was expected for two reasons: firstly, the thermal contact between thermocouple and sample was not perfect due to the difference in thickness between weld joint and the hole; moreover, the temperature was not measured exactly at the weld joint due to the presence of the $3 \mathrm{~mm}$ plait. Secondly, in the present experiment the sample was submitted to the heating loads only on the front side. As already explained, such heating configuration is responsible of a temperature gradient between front side where the temperature was measured by the MC pyrometer and the back side where the thermocouple is installed. To precise the difference, we estimated experimentally and theoretically the error in the temperature measurement as a function of emissivity $\epsilon$. In Fig. 7, we show the temperature deviation $T(\epsilon)-T(\epsilon=0.32)$ with different experimental setting of $\epsilon, 0.25<\epsilon<0.35$, for two different temperatures $(1330 \mathrm{~K}$ on the top and 2100 $\mathrm{K}$ on the bottom) measured at $\epsilon=0.32$.

Moreover, we have estimated the dependence of the relative error made on the temperature as a function of $\Delta \epsilon$ by using the following relation

$$
\frac{e_{T}}{T} \approx\left|\frac{\lambda T}{c_{2}}\right| \frac{e_{\epsilon}}{\epsilon} \approx 10^{-4} T \frac{e_{\epsilon}}{\epsilon},
$$

where lambda is the pyrometer working wavelength, $\mathrm{C}_{2}$ is Planck's second radiation constant, $e_{\epsilon}$ is the emissivity error. As shown in Fig. 7, calculated and measured temperature are in good agreement. One can note that the relative error is directly proportional to the sample temperature, this is the reason of the change of slope of relative error as a function of emissivity for the two different temperatures.

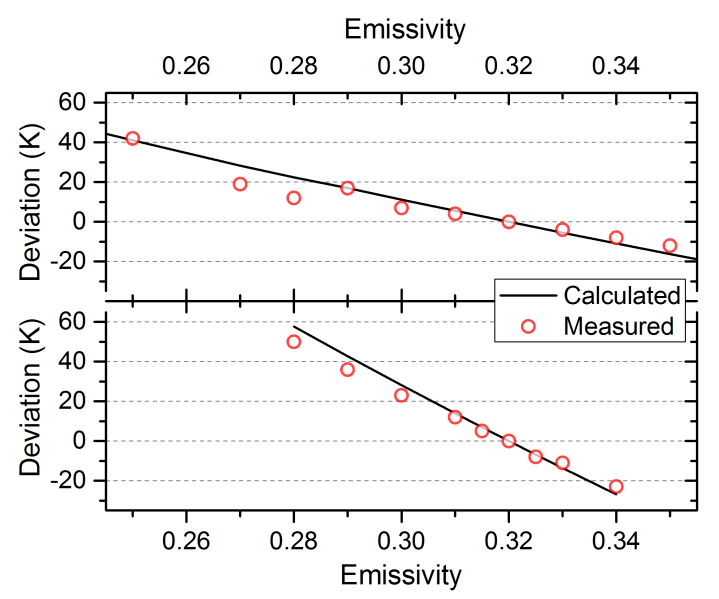

FIG. 7. Measured (dots) and calculated (lines) temperature deviation $T(\epsilon)-T(\epsilon=0.32)$ with $0.25<\epsilon<0.35$ for two different temperatures $(1330 \mathrm{~K}$ top panel and $2100 \mathrm{~K}$ bottom panel) measured at $\epsilon=0.32$

\section{B. Thermal gradient during laser heating}

We have discussed in the previous section about the issue of possible thermal gradients in the sample during the laser annealing sequence. In order to experimentally investigate these thermal gradients, we have used a fast and high definition thermal camera (FLIR x8500sc) to record the surface temperature of the sample during some annealing sequences. The camera, based on a $\mathrm{InSb}$ detector, operates in the range $3-5 \mu \mathrm{m}$ and is calibrated by the manufacturer from ambient to $2273 \mathrm{~K}$. Images were taken with a $50 \mathrm{~mm}$ objective through a ZnSe viewport, which transmission was calibrated on a black body source, at a frame rate of $250 \mathrm{~Hz}$. The absolute temperature reported in these measurements should then been taken with caution since the emissivity is temperature dependent in the operating spectral range, nevertheless the relative gradients of interest are accurate.

We give on Fig. 8 example of images extracted from a heating sequence up to $1673 \mathrm{~K}$ and the following cooling phase. We have plotted on Fig. 9 the thermal profile along the line shown on Fig. 8, corresponding to the temperature plateau of $1673 \mathrm{~K}$. The temperature deviation on the sample surface is $+/-15 \mathrm{~K}$ which in fact corresponds to the sensor accuracy (given as $+/-2 \%$ of the temperature reading by the manufacturer). We can also observe negligible thermal gradient along the surface which is not exposed to the laser flux. This lead us to conclude that in this configuration the laser heating technique does not introduce significant thermal gradients, in accordance with the numerical simulations.

\section{Ramp temperature}

The use of high power laser as heating source is very useful to submit samples to fast heating ramps. Panel $a$ of Fig. 10 shows some examples of linear heating ramps from 873 to $1600 \mathrm{~K}$ for six laser heating duration $(0.1,0.3$, $0.5,1,4.5$, and $9.5 \mathrm{~s}$ ) obtained using the PID controller. Fig. $10 b$ presents the heating ramp in $\mathrm{K} / \mathrm{s}$ as a function of irradiation time obtained by derivation of Fig. 10a. The temperature was measured via the MC pyrometer as shown in Fig. 5. The heating ramps are linear except in the case of $0.1 \mathrm{~s}$ irradiation where the ramp starts at $\sim 7000 \mathrm{~K} / \mathrm{s}$ and reaches $4000 \mathrm{~K} / \mathrm{s}$ at the end of laser irradiation (i.e. $0.1 \mathrm{~s}$ ). In the other cases, the ramps are linear for the entire heating time with fluctuations lower than $10 \%$ of the initial ramp. Linear ramps range from 2400 to $73 \mathrm{~K} / \mathrm{s}$ for 0.3 and $10 \mathrm{~s}$ heating time, respectively. The heating ramp strongly depends on the used sample and faster heating ramps could be attained for thinner samples. Moreover, we stress that in the present experiments, we have never used the full laser power $(1500 \mathrm{~W})$ that can certainly reduce the heating time. At the moment, the acquisition time of the temperature software is $10 \mathrm{~ms}$ and it represents the main limitation to measure faster heating ramps. We point out that both PID and 


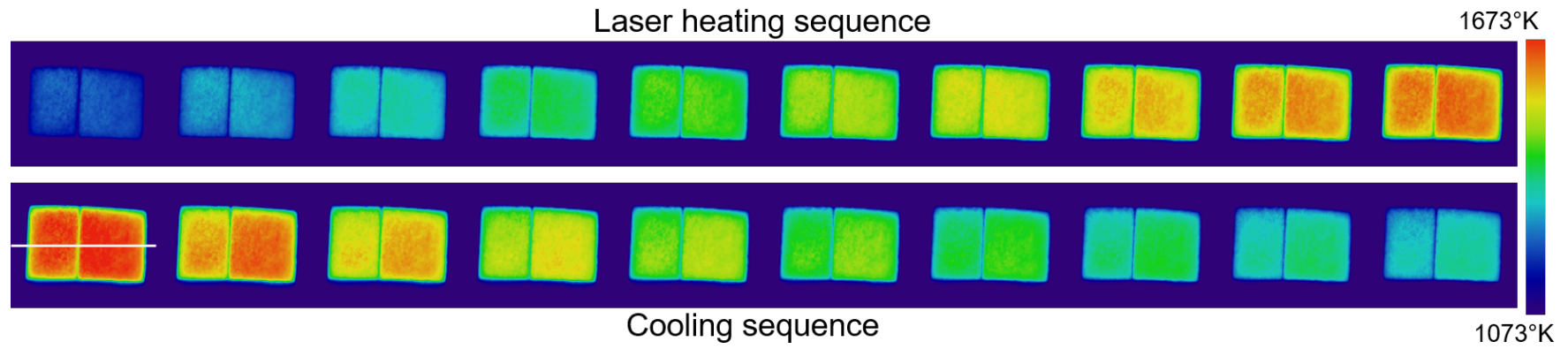

FIG. 8. Observation of a heating and cooling sequence with a thermal camera. The time between each frame in this sequence is $0.5 \mathrm{~s}$. The sample, a $4 \times 4 \times 5 \mathrm{~mm}^{3} \mathrm{~W}$ block in the configuration described previously, is observed through a view-port of the chamber at 45 degrees incidence with the laser.

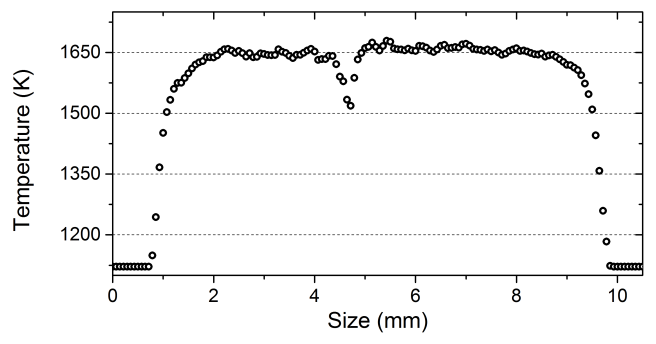

FIG. 9. Temperature profile of the tungsten sample after 5 $\mathrm{s}$ of laser heating along the line shown in Figure 8 (the first frame of the bottom panel).

MC pyrometer have sampling times of $1 \mathrm{~ms}$ and $100 \mu \mathrm{s}$ respectively.

\section{APPLICATION}

As presented above, the laser facility ChauCoLase can be used to submit materials to extreme heat load with a large flexibility in terms of sample size, maximum temperature, spatial and temporal heating profiles. ChauCoLase was employed for the laser treatment of different materials, like graphite, $\mathrm{W}$, and $\mathrm{UO}_{2}{ }^{29}$. In the present paper, we show a set of experiments performed on $\mathrm{W}$ to study and characterize its recrystallization kinetics. To determine the recrystallization fraction, post-mortem hardness measurements (Vickers hardness test) and Electron BackScatter Diffraction (EBSD) microscopy were performed before and after annealing. From hardness measurements, recrystallization fraction $(\mathrm{X})$ was calculated from:

$$
X(t)=\frac{H V_{0}-H V(t)}{H V_{0}-H V_{\text {recr }}}
$$

where $H V_{0}$ and $H V_{\text {recr }}$ are the Vickers hardness values of the material prior to and after $100 \%$ of recrystallization.

EBSD was used to determine the grain size distribution, the density of high and low angle grain boundaries
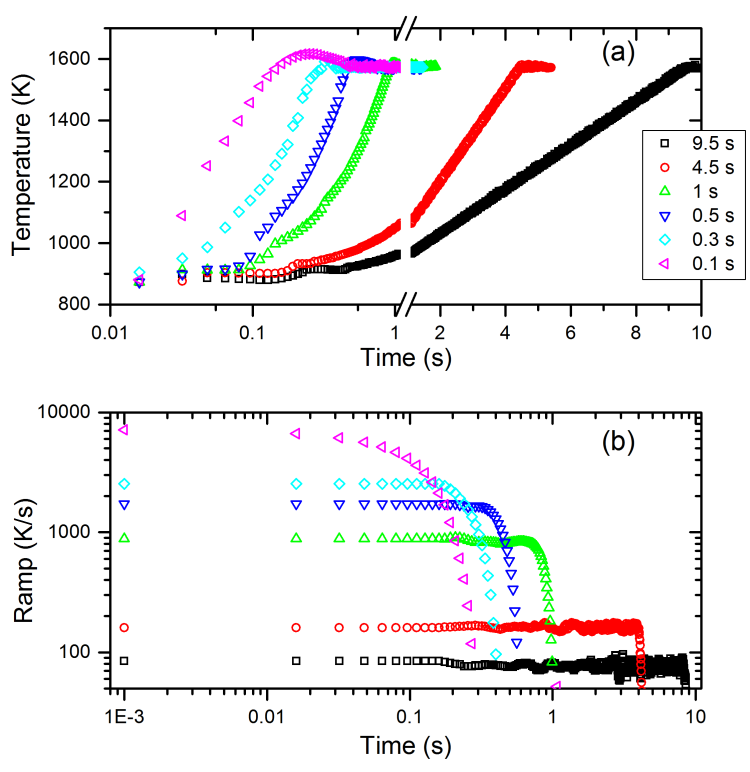

FIG. 10. Panel a: Temperature increase of a tungsten sample from $873 \mathrm{~K}$ to $1573 \mathrm{~K}$ as a function of laser heating duration $(0.1,0.3,0.5,1,4.5$, and $9.5 \mathrm{~s})$. Panel $b$ : Heating ramps as a function of time for data shown in panel $a$.

such as the crystallographic texture. All these parameters are known to highly affect recovery and recrystallization kinetics ${ }^{1}$. During primary recrystallization, the new grains grow at the expanse of the strain hardened microstructure, the driving force being the stored energy associated to dislocations and sub-grain boundaries. This stored energy induces an intragranular crystal orientation gradient, that can be measured using $\mathrm{EBSD}^{30}$, as shown for a rolled $\mathrm{W}$ in Fig. 11. In the case of tungsten for which cold or warm deformation microstructures are mostly composed of subgrain boundaries (i.e. the lowangle boundary, LAB), an efficient approximation to estimate the recrystallized fraction is to assume that grains with no LABs are recrystallized grains.

The time evolution of the $\mathrm{W}$ recrystallized frac- 
tion for a fixed temperature was described using the Johnson-Mehl-Avrami-Kolmogorov (JMAK) ${ }^{1,31}$ isothermal model:

$$
X(t)=1-\exp \left(-B^{n_{A}}\left(t-t_{i n c}\right)^{n_{A}}\right)
$$

where $B$ is a parameter, $n_{a}$ is the Avrami exponent, and $t_{\text {inc }}$ is the incubation time. All these parameters follow an Arrhenius-type dependence on temperature.

W-sample were cut with Electrical Discharge Machining (EDM) technique and it is well known that the surface integrity of materials machined by EDM is typically poor $^{32}$. The presence of significant carbon and oxides contamination at surface after machining ${ }^{33,34}$ are responsible of an evolution of optical properties of materials. Moreover, the contamination depends on the physicalchemical conditions present during EDM machining and it can change from one sample to another cut from the same batch. For such a reason, the exact determination of the emissivity after cutting is a critical issue when using optical systems to determine the sample temperature. On the other hand, the emissivity measurement of each sample would be difficult and time consuming since surface state and contamination evolve during the initial phase of the annealing. To circumvent this issue each sample was submitted to a "cleaning" laser annealing sequence. Samples were heated to $1400 \mathrm{~K}$ for 300 seconds. Fig. 12 shows a sample before and after the cleaning sequence. We stress that the cleaning sequence of 300 second should induce a negligible change (few per mil) on the recrystallization fraction. As shown by Alfonso $^{23}$ (Table 6 at page 61 ), a warm-rolled tungsten presents only a $\mathrm{X}=14 \%$ after 950 hours at $1423 \mathrm{~K}$. Once the sample cleaned, the annealing temperature $\mathrm{T}_{a}$ is reached for the time $t_{a}$. Laser is stopped after the sequence and the sample is cooled down by natural conduction/radiation. We show in Fig. 13 the temperature profile recorded on the center at the laser-exposed face of a sample annealed to $2073 \mathrm{~K}$ for 600 seconds. Similar laser

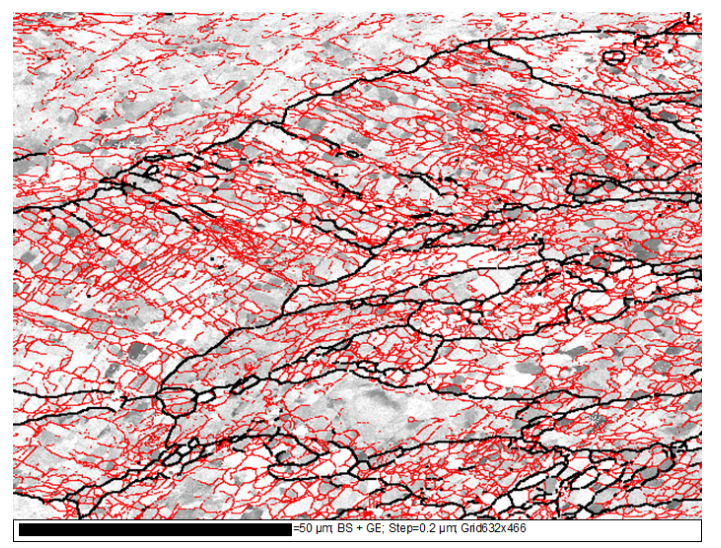

FIG. 11. Inital microstructure of a rolled W (from EBSD measurement) : High Angle Boundaries (Black lines), Low Angle Boundaries (Red line), Substructures (grey level contrast band slope).

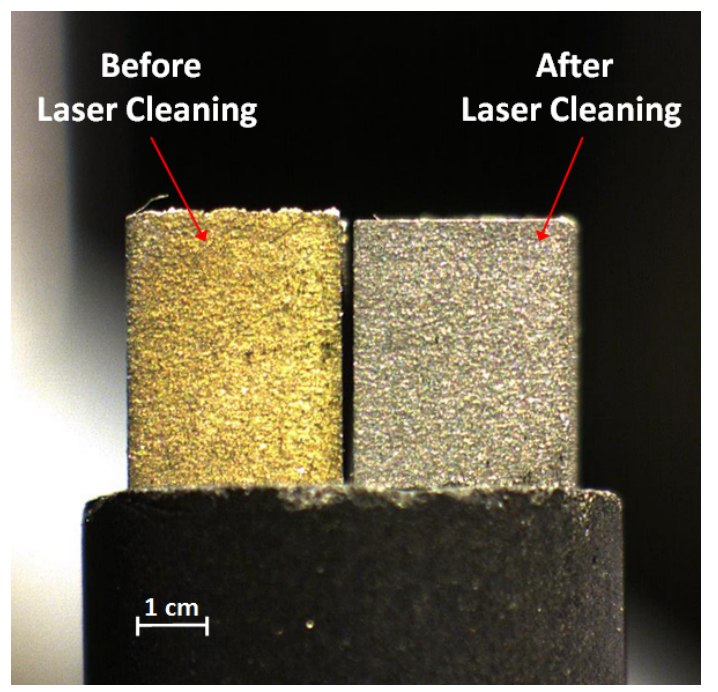

FIG. 12. Picture of $\mathrm{W}$ samples before and after the cleaning annealing sequence.

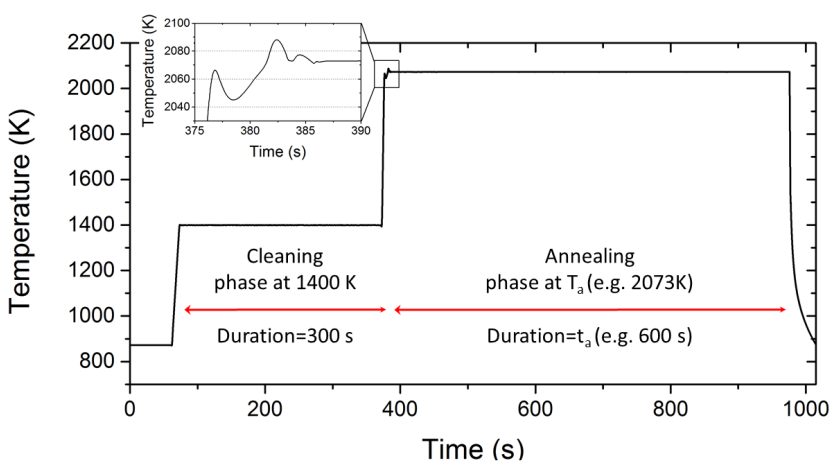

FIG. 13. Typical laser heating sequence used in this work. The zoom-in shows the overshoot of the initial phase of annealing sequence.

heating sequences are employed in this work by changing $\mathrm{T}_{a}$ and $\mathrm{t}_{a}$. One can note that an overshoot is present in the initial phase of annealing sequence: zoom-in of Fig. 13 shows the longest $(\sim 5 \mathrm{~s})$ and most intense $( \pm 35$ $\mathrm{K}$ ) overshoot measured in our experiments. Typical overshoots present a temperature deviation from $\mathrm{T}_{a}$ of $12 \mathrm{~K}$ for $0.5 \mathrm{~s}$. Overshoot is caused by a "slow" response of PID controller and its intensity depends mainly on two parameters: heating rate and $\mathrm{T}_{a}$. It could be reduced by a finetuning of PID parameters (in particular I parameter) for each annealing temperature. Nevertheless, in the present work we did not pushed forward with the tuning of PID parameters since $t_{a}>>t_{\text {overshoot }}$ and temperature error $\Delta T_{a} \sim T_{\text {overshoot }}$. In other words, we considered negligible the recrystallization fraction during the overshoot with respect to the annealing sequence. Fig. 14 presents a typical recrystallization kinetics of $\mathrm{W}$ measured through Vickers hardness test. Here, the samples were annealed at $\mathrm{T}_{a}=1873 \mathrm{~K}$ for times ranging between 296 and $3000 \mathrm{~s}$. 


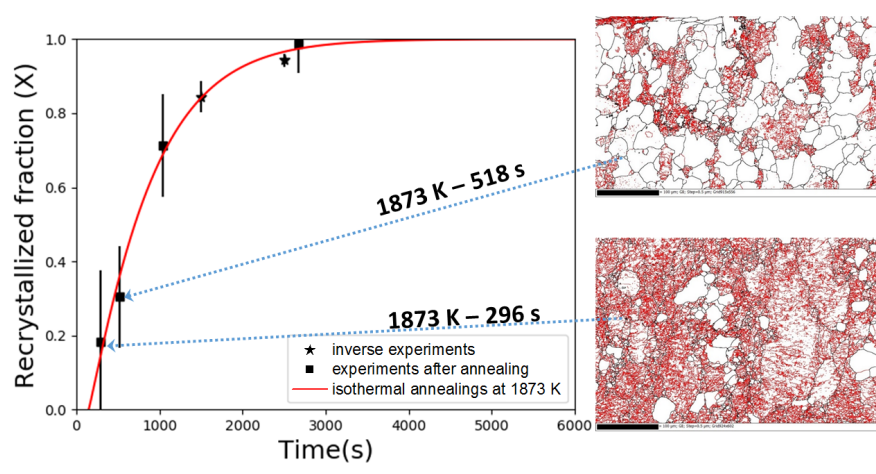

FIG. 14. Recrystallization kinetics of $\mathrm{W}$ annealed at $1873 \mathrm{~K}$. On the right, EBSD images for two different annealing times (296 and $598 \mathrm{~s}$ ).

The $\mathrm{W}$ microstructure and initial microstructural state are assessed with Electron Back Scattering Diffraction (EBSD) analysis as shown on the right side of Fig. 14 for two different annealing times (296 and $598 \mathrm{~s}$ ). In this figure, the microstructure is partly recrystallized consisting in a mixture of recrystallized grains and deformed grains (recovered matrix). As expected the higher the annealing time the higher the area of defect-free grains (no stored energy). Let us note that the recrystallization fraction measured from hardness data and EBSD can differ significantly since both recovery and rerystallization plays on hardness. It may even be difficult to state from EBSD if a defect-free grain is the consequence of recrystallization or recovery. The transformation in grain morphology from rolling induced elongated grains to equiaxed grain is a good indicator of the recrystallization process. Therefore Fig. 14 clearly pointed out that a recrystalization process happens during annealing of $\mathrm{W}$ at $1873 \mathrm{~K}$.

\section{CONCLUSIONS}

In this work, we presented a high power laser facility developed to submit materials to extreme heat loads. Such facility is of particular interest to investigate microstructure evolution - such as recrystallization - during a heat treatment at a very high temperature. The laser remote heating presents different advantages with respect to other annealing techniques: (1) it is a contactless technique which can easily drive materials to extreme temperatures with very high spatial precision and temporal control; (2) a fast and homogenous heating rate can be reached, which minimizes sample microsctrure evolution before the annealing temperature is reached; (3) with focused laser beam application, the sample being tested essentially acts as its own containment vessel, avoiding any contaminants, and virtually without affecting the surroundings because only a small part of the material is being treated; (4) due to the possibility to have optical access to the sample, laser heating can be combined to several non-contact diagnostics such as infrared imaging to derive meaningful thermo physical properties avoiding interferences that would normally occur with direct contact measurements of temperature.

We presented some applications focusing our attention to a set of experiments performed on W to study and characterize its recrystallization kinetics at elevated temperature.

\section{ACKNOWLEDGMENTS}

We acknowledge the support of Fédération de Recherche sur La Fusion par Confinement Magnétique.

This work has been partly funded under the CEA PJIPG's program.

${ }^{1}$ F. J. Humphreys and M. Hatherly. Recrystallization and related annealing phenomena. Pergamon, Oxford, OX, UK ; Tarrytown, N.Y., U.S.A, 1st ed edition, 1995.

${ }^{2}$ P.A. Beck. In Gordon Smith and Breach, editors, Sorby Centennial Symposium on the History of Metallurgy, volume 27, page 313, New York, 1963. Metallurgy Society Conference.

${ }^{3}$ Jian Wang, Jun Li, Xinfeng Wang, Xiaochuan Mi, and Shengen Zhang. Rapid heating effects on grain-size, texture and magnetic properties of $3 \% \mathrm{Si}$ non-oriented electrical steel. Bulletin of Materials Science, 34(7):1477-1482, December 2011.

${ }^{4}$ Saeed Nobakht and Mohsen Kazeminezhad. Electrical annealing of severely deformed copper: microstructure and hardness. International Journal of Minerals, Metallurgy, and Materials, 24(10):1158-1168, October 2017.

${ }^{5}$ H. Ghiabakloo and M. Kazeminezhad. Rapid annealing of severely deformed low carbon steel in subcritical temperature range. Metals and Materials International, 23(5):984-993, September 2017.

${ }^{6}$ Paulo Pedrosa, Jean-Marc Cote, Nicolas Martin, Mohammad Arab Pour Yazdi, and Alain Billard. In situ electrical resistivity measurements of vanadium thin films performed in vacuum during different annealing cycles. Review of Scientific Instruments, 88(2):025105, February 2017.

${ }^{7}$ J. M. O'Connor, H. S. Hier, and R. M. Ketchum. Annealing furnace for III-V semiconductor devices. Review of Scientific Instruments, 57(2):206-208, February 1986.

${ }^{8}$ Ming Xie, Chee Huei Lee, Jiesheng Wang, Yoke Khin Yap, Paola Bruno, Dieter Gruen, Dileep Singh, and Jules Routbort. Induction annealing and subsequent quenching: Effect on the thermoelectric properties of boron-doped nanographite ensembles. Review of Scientific Instruments, 81(4):043909, April 2010.

${ }^{9}$ R. A. McMahon, D. G. Hasko, and H. Ahmed. Electron beam system for rapid isothermal annealing of semiconductor materials and devices. Review of Scientific Instruments, 56(6):1257-1261, June 1985.

${ }^{10}$ Sadhan Chandra Das, Abhijit Majumdar, Sumant Katiyal, T. Shripathi, and R. Hippler. Development of fast heating electron beam annealing setup for ultra high vacuum chamber. Review of Scientific Instruments, 85(2):025107, February 2014.

${ }^{11}$ Florent Lefevre-Schlick, Xiang Wang, and David Embury. The production of fine-scale microstructures by rapid annealing. $M a-$ terials Science and Engineering: A, 483-484:258-261, June 2008.

$12 \mathrm{~J}$ Dutta Majumdar and I Manna. Laser material processing. International Materials Reviews, 56(5-6):341-388, November 2011.

${ }^{13}$ Eiji Fujimoto, Masatomo Sumiya, Tsuyoshi Ohnishi, Mikk Lippmaa, Masaki Takeguchi, Hideomi Koinuma, and Yuji Matsumoto. Development of a new laser heating system for thin film growth by chemical vapor deposition. Review of Scientific Instruments, 83(9):094701, September 2012. 
${ }^{14}$ S. P. Murzin. Local Laser Annealing for Aluminium Alloy Parts. LASERS IN ENGINEERING, 33(1-3):67-76, 2016.

${ }^{15}$ X. Duan, H. Huneus, T. Kochmann, K. Leuridan, R. Kaczmarek, and F. Protat. Effect of annealing temperature and heating rate on the magnetic and mechanical properties of electrical steel. Journal of Magnetism and Magnetic Materials, 160:133-135, July 1996.

${ }^{16}$ A. M. Guerenu, F. Arizti, M. D. Fuentes, and Gutierrez I. Acta Materialia, 52:3657, 2004.

${ }^{17}$ M. Merola, F. Escourbiac, R. Raffray, P. Chappuis, T. Hirai, and A. Martin. Overview and status of iter internal components. Fusion Engineering Design, 89(7-8):890-895, 2014.

${ }^{18}$ T. Hirai, S. Panayotis, V. Barabash, C. Amzallag, F. Escourbiac, A. Durocher, M. Merola, J. Linke, Th. Loewenhoff, G. Pintsuk, M. Wirtz, and I. Uytdenhouwen. Use of tungsten material for the iter divertor. Nuclear Materials and Energy, 9:616 - 622, 2016.

${ }^{19}$ K. Ezato, S. Suzuki, Y. Seki, K. Mohri, K. Yokoyama, F. Escourbiac, T. Hirai, and V. Kuznetcov. Progress of iter full tungsten divertor technology qualification in japan. Fusion Engineering and Design, 98-99:1281 - 1284, 2015. Proceedings of the 28th Symposium On Fusion Technology (SOFT-28).

${ }^{20}$ T. Hirai, F. Escourbiac, V. Barabash, A. Durocher, A. Fedosov, L. Ferrand, T. Jokinen, V. Komarov, M. Merola, S. CarpentierChouchana, N. Arkhipov, V. Kuznetcov, A. Volodin, S. Suzuki, K. Ezato, Y. Seki, B. Riccardi, M. Bednarek, and P. Gavila. Status of technology R\&D for the ITER tungsten divertor monoblock. Journal of Nuclear Materials, 463:1248-1251, August 2015.

${ }^{21}$ Gerald Pintsuk. Characterization of ITER tungsten qualification mock-ups exposed to high cyclic thermal loads. Fusion Engineering and Design, page 5, 2015.

${ }^{22}$ A. Durif, M. Richou, G. Kermouche, M. Lenci, and J-M. Bergheau. Impact of tungsten recrystallization on ITER-like components for lifetime estimation. Fusion Engineering and Design, 138:247-253, January 2019.

${ }^{23}$ A. Alfonso Lopez, W. Pantleon, D. Juul Jensen, and G. Luo. Thermal stability of warm-rolled tungsten. PhD thesis, Technical University of Denmark, Section of Materials and Surface Engineering, Department of Mechanical Engineering, June 2015.

${ }^{24}$ M. Wirtz, J. Linke, T. Loewenhoff, G. Pintsuk, and I. Uytden- houwen. Transient heat load challenges for plasma-facing materials during long-term operation. Nuclear Materials and Energy, 12:1-8, 2016.

${ }^{25} \mathrm{~S}$. Panayotis and et al. Self-castellation of tungsten monoblock under high heat flux loading and impact of material properties. Nuclear Materials and Energy, 12:200-204, 2016.

${ }^{26}$ Marco Minissale, Cedric Pardanaud, Régis Bisson, and Laurent Gallais. The temperature dependence of optical properties of tungsten in the visible and near-infrared domains: an experimental and theoretical study. Journal of Physics D: Applied Physics, 50(45):455601, oct 2017

${ }^{27}$ J. C. DeVos. Physica, XX:690-714, 1954.

${ }^{28}$ C. Cagran, M. G. Pottlacher, Rink, and W. Bauer. Spectral emissivities and emissivity x-points of pure molybdenum and tungsten. International Journal of Thermophysics, 27:1001, December 2005.

${ }^{29}$ T. Vidal, L. Gallais, J. Faucheux, H. Capdevila, J. Sercombe, and Y. Pontillon. Overview and status of iter internal components. Accepted for Journal of Nuclear Materials, $\mathrm{xx}(\mathrm{xx})$ : $\mathrm{xxx}-$ $\mathrm{xxx}, 2019$.

${ }^{30}$ P. Baral, M. Laurent-Brock, G. Guillonneau, J.M. Bergheau, J.L. Loubet, and G. Kermouche. In situ characterization of aa1050 recrystallization kinetic using high temperature nanoindentation testing. Materials and Design, 152:22-29, 2018.

${ }^{31}$ A. Alfonso, D. Juul Jensen, and W. Luo, G.-N.and Pantleon. Recrystallization kinetics of warm-rolled tungsten in the temperature range $1150-1350{ }^{\circ} \mathrm{C}$. Journal of Nuclear Materials, 455(1):591-594, 2014.

${ }^{32}$ James Murray. Damage, Contamination and Surface Treatment of Electrical Discharge Machined Materials. PhD thesis, University of Nottingham, February 2014.

${ }^{33}$ J.W. Murray, M.W. Fay, M. Kunieda, and A.T. Clare. Tem study on the electrical discharge machined surface of single-crystal silicon. Journal of Materials Processing Technology, 213(5):801809, May 2013.

${ }^{34}$ Tadeusz Leppert. A review on ecological and health impacts of electro discharge machining (EDM). In XIII INTERNATIONAL CONFERENCE ELECTROMACHINING 2018, page 020014, Bydgoszcz, Poland, 2018. 